\title{
Modelling Time-dependent Release Kinetics in Stent-based Delivery
}

\author{
Ramprosad Saha ${ }^{1}$ and Prashanta Kumar Mandal ${ }^{2 *}$ \\ ${ }^{1}$ Department of Mathematics, Suri Vidyasagar College (Affiliated by University of Burdwan), Suri-731101, West Bengal, India; \\ ${ }^{2}$ Department of Mathematics, Visva-Bharati University, Santiniketan-731235, West Bengal, India
}

\begin{abstract}
Background and objective: The present study deals with a computational model of the transport and retention of drug within the arterial wall eluted from a drug-eluting stent, to enhance our understanding of the performance of this device. We considered a two-species model (free and bound) incorporating a reversible reaction to describe drug interactions with the constituents of the arterial wall. An axisymmetric model of drug delivery from a pair of stent struts has been developed, where the transport of free drug is modelled as an unsteady reaction-diffusion process, while the bound drug, assuming complete immobilization in the tissue, is modeled as an unsteady reaction process. The model also took into account a second-order binding process that describes a saturating reversible binding and time-dependent release kinetics of the drug-eluting stent. Considering that diffusion takes place over a tortuous path in a porous media, the effects of porosity and tortuosity on diffusion cannot be ruled out from this present investigation.
\end{abstract}

Methods: An explicit finite-difference scheme is leveraged to tackle numerically the governing equations of motion, together with the physiologically realistic boundary conditions.

Results: The quantitative effects of significant factors, such as Peclet number $\left(P e_{T}\right)$, Damköhler number (Da), tortuosity, porosity, interstrut distance and time-dependent release kinetics of drug-eluting stent, on the distribution and retention of drug are determined graphically.

Conclusions: Predicted results are consistent with several existing results in the literature, which certainly validates the applicability of the model considered.

Introduction

Arterial stenting, acting as a supporting scaffold, has revolutionized the treatment of coronary artery disease, as it reopens the occluded vessel and restores the normal flow of blood. The bare metal stents $\left(B M S^{s}\right)$, while revolutionary at the time, were soon rendered unsatisfactory due to their inability to prevent in-stent restenosis. The next wave of arterial stents coated by drug-the so-called drug-eluting stents $\left(D E S^{S}\right)$ raised all sorts of questions by releasing antiproliferative

Keywords: Two-species model; Drug-eluting stent; Reaction-diffusion process; Time-dependent release kinetics; Interstrut distance; Porosity; Tortuosity.

Abbreviations: BMS, bare metal stent; CAD, coronary artery disease; DES, drugeluting stent; ISR, in-stent restenosis; PCI, percutaneous coronary intervention.

Received: January 06, 2018; Revised: February 27, 2018; Accepted: March 02, 2018

*Correspondence to: Prashanta Kumar Mandal, Department of Mathematics, VisvaBharati University, Santiniketan-731235, West Bengal, India. Tel: +91-3463-261029; Fax: +91-3463-261029; E-mail: pkmind02@yahoo.co.uk

How to cite this article: Saha R, Mandal PK. Modelling Time-dependent Release Kinetics in Stent-based Delivery. J Explor Res Pharmacol 2018;3(2):61-70. doi: 10.14218/ JERP.2018.00001 agents in a controlled manner into the injured site to reduce restenosis rates. ${ }^{1-9}$ Drug-eluting stents are now the primary choice of percutaneous coronary interventions $\left(P C I^{s}\right)$ in millions of patients, but questions regarding their longevity and safety still arise.

Some experimental studies have been carried out in the recent past, with the aim of quantifying the capability of this device to reduce in-stent restenosis after stent implantation. ${ }^{10-12}$ Lovich et $a l .{ }^{10}$ studied the behaviour of heparin in explanted arteries and concluded that the presence of binding sites changes along the transmural direction, being higher in the endothelium and lower in the adventitia. Lovich and Edelman studied the effects of specific binding sites inside the arterial wall on the drug uptake, ${ }^{13}$ where the presence of specific binding site action was modelled using the reversible chemical reaction. Sakharov et al. ${ }^{14}$ disregarded the convective effects on the transport of free drug. Hwang et al. ${ }^{15}$ predicted the free as well as bound drug concentrations by solving for distribution of free drug and then using a multiplicative factor (partition approach) to predict the concentration of bound drug. Migliavacca et al. ${ }^{16}$ studied the drug release pattern in vascular wall from drug-eluting stents using a single species approach along with a partition coefficient approach to relate the free and the 


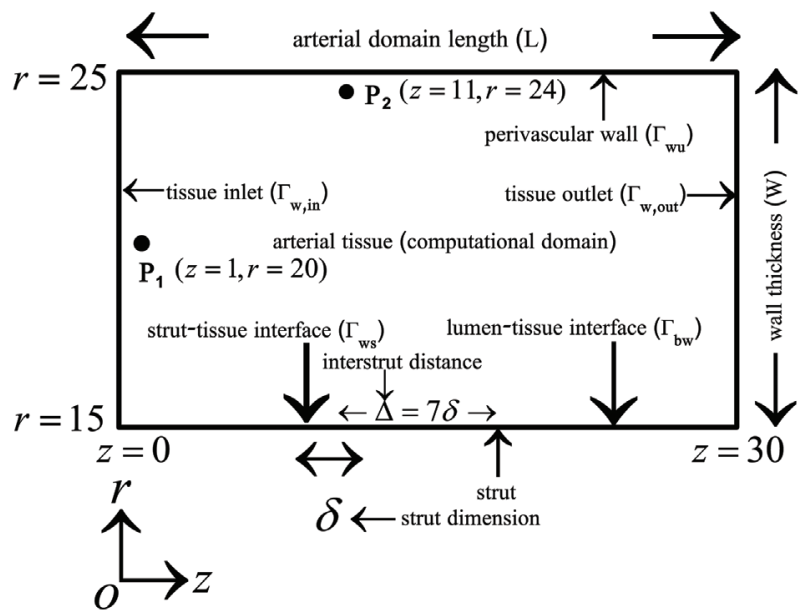

Fig. 1. Schematic diagram of the computational domain.

bound drug concentrations. Borghi et al. ${ }^{17}$ opined that the inclusion of reversible binding leads to delayed release and that the erosion of polymer affects the drug release from a single strut. Horner et al. ${ }^{18}$ considered a two-species drug delivery model including reversible binding sites, and their model predicted that a single species drug delivery model cannot accurately predict the distribution of bound drug. They also concluded that a two-species approach that includes reversible binding is the way forward for future stentbased drug delivery systems.

The main objective of this study was to advance the aforementioned work with a two-species model of drug transport eluted from a pair of struts, where the transport of free drug is governed by reaction-diffusion process and that of bound drug, assuming complete immobilization in the tissue, by reaction process. Following Tzafriri et al., ${ }^{19}$ a second-order dynamic model that describes a saturating reversible binding process by treating bound drug as a dynamic variable has been taken into account to explore drug interaction with cells of the arterial wall. In most of the studies cited above, transient drug release has been modelled as a uniform release, which is unrealistic and not representative of actual stent-based delivery. Instead, a simple time-dependent Dirichlet boundary condition is applied on the surface of the struts. ${ }^{20-24} \mathrm{Ar}-$ terial properties, such as porosity and tortuosity, dictate the transport of drugs within the arterial tissue.

When an endovascular drug-eluting stent is implanted, it has major impact on the structure of the arterial wall, eventually influencing the overall rates of diffusion through tissues. ${ }^{25}$ For diffusion in a porous material, the effective diffusion coefficient is assumed to depend on two factors viz porosity (a dimensionless parameter, which is the ratio of pore volume to the total material volume) and diffusion path tortuosity (ratio of the actual pore length to the distance between its ends; i.e. arc-chord ratio) ${ }^{26}$ - these parameters change the free diffusivity of the drug eluted from a pair of struts. ${ }^{27}$ Thus, the effects of porosity and tortuosity on the diffusivity of drug have also been accounted for in this present investigation.

\section{Governing equations and boundary conditions}

The freely-transported drug, referred to as 'free drug', is denoted by $c_{f}$ and the drug, completely immobilized in the tissue, is referred to as 'bound drug', which is denoted by $c_{b}$. The interconversion of drug between the unbound plasma phase and the bound phase of tissue binding sites is controlled by a second-order chemical reaction. The computational domain is comprised of a long axial section of length $L$ and the wall thickness is taken to be 10 times the strut height $(\delta)$. The axis of symmetry is taken along the centerline of the artery (Fig. 1). The transport of free drug eluted from a pair of struts is governed by unsteady reaction-diffusion process (Eq. 1 ) and that of the bound drug is represented by unsteady reaction process (Eq. 2).

Symmetry boundary conditions for both the free and the bound drugs are applied at the proximal $\left(\Gamma_{t i}\right)$ and the distal $\left(\Gamma_{t o}\right)$ walls (Eq. 3). ${ }^{28,29}$ Impermeable boundary condition for bound drug is assumed at the perivascular wall $\left(\Gamma_{t p}\right)$, lumen-tissue $\left(\Gamma_{b t}\right)$ and struttissue $\left(\Gamma_{s t}\right)$ interfaces (Eq. 4). For the free drug, perfect sink condition is imposed at the perivascular end (Eq. 5). Since a proper boundary condition for the free drug at lumen-tissue interface $\left(\Gamma_{b t}\right)$ is not readily apparent, we considered two opposing extremes, either that flowing blood is extremely efficient at washing out muraladhered drug, modelled as a zero-concentration interface condition, or that mural-adhered drug is insensitive to flowing blood, modelled as zero-flux boundary condition (Eq. 6)..$^{30-31}$ Instead of modelling uniform release of drug from a pair of struts, we assumed simple time-dependent release kinetics with a flux condition (Eq. 7). ${ }^{13,20}$ Therefore, the governing equations representing the transport of free as well as bound drugs together with their appropriate boundary conditions are as follows:

$$
\begin{gathered}
\frac{\partial c_{f}}{\partial t}=D_{T}\left[\frac{\partial^{2} c_{f}}{\partial r^{2}}+\frac{1}{r} \frac{\partial c_{f}}{\partial r}+\frac{\partial^{2} c_{f}}{\partial z^{2}}\right] \\
-\left[k_{a} c_{f}\left(B_{M}-c_{b}\right)-k_{d} c_{b}\right], \\
\frac{\partial c_{b}}{\partial t}=\left[k_{a} c_{f}\left(B_{M}-c_{b}\right)-k_{d} c_{b}\right], \\
\frac{\partial c_{f}}{\partial z}=0=\frac{\partial c_{b}}{\partial z} \text { on } \Gamma_{\mathrm{ti}} \text { and } \Gamma_{\mathrm{to}}, \\
\frac{\partial c_{b}}{\partial r}=0 \text { on } \Gamma_{\mathrm{tp}} \text { and } \Gamma_{\mathrm{tl}}\left(=\Gamma_{\mathrm{bt}} \cup \Gamma_{\mathrm{st}}\right), \\
c_{f}=0 \text { on } \Gamma_{\mathrm{tp}}, \\
c_{f}=0 \text { or } \frac{\partial c_{\mathrm{f}}}{\partial \mathrm{r}}=0 \text { on } \Gamma_{\mathrm{bt}}, \\
J_{b}(t)=\sqrt{\frac{D_{c} c_{0}^{2}}{\pi t}} \text { on } \Gamma_{\mathrm{st}},
\end{gathered}
$$

where $z$ and $r$ coordinates are along the axial and the radial directions respectively. $k_{a}$ and $k_{d}$ are the rates of association and dissociation constants respectively. $B_{M}$ is the net tissue binding capacity. $D_{c}$ and $c_{0}$ are the diffusion coefficient and initial concentration in coating respectively.

Here, $D_{T}$, the true diffusivity of the free drug can be written as: ${ }^{27,32}$

$$
D T=\left(1+\frac{B_{M}}{R_{d}}\right) \times D_{e f f},
$$

where 


$$
D_{\text {eff }}=\frac{\varepsilon}{\tau} \times D_{\text {free }} .
$$

$\varepsilon$ and $\tau$ are the porosity and the tortuosity of the wall material respectively. $D_{\text {free }}$ and $D_{\text {eff }}$ are the coefficients of free and effective diffusivity respectively. $R_{d}\left(=k_{d} / k_{a}\right)$ is the equilibrium dissociation constant.

We now introduce nondimensional variables as

$$
x=\frac{r}{\delta}, \bar{z}=\frac{z}{\delta}, \bar{t}=\frac{t V_{y}}{\delta}, \bar{c}_{f}=\frac{c_{f}}{c_{0}}, \bar{c}_{b}=\frac{c_{b}}{B_{M}},
$$

where $V_{y}$ is the transmural filtration velocity.

Under these assumptions, the equations (1-7) take their respective nondimensional forms (dropping tilde) as

$$
\begin{gathered}
\frac{\partial c_{f}}{\partial t}=\frac{1}{P e_{T}}\left[\frac{\partial^{2} c_{f}}{\partial x^{2}}+\frac{1}{x} \frac{\partial c_{f}}{\partial x}+\frac{\partial^{2} c_{f}}{\partial z^{2}}\right] \\
-\frac{D a}{P e_{T}}\left[c_{f}\left(1-c_{b}\right)-\varepsilon_{1} c_{b}\right], \\
\frac{\partial c_{b}}{\partial t}=\frac{\varepsilon_{2} D a}{P e_{T}}\left[c_{f}\left(1-c_{b}\right)-\varepsilon_{1} c_{b}\right], \\
\frac{\partial c_{f}}{\partial z}=0=\frac{\partial c_{b}}{\partial z} \text { on } \Gamma_{\mathrm{ti}} \text { and } \Gamma_{\mathrm{to}}, \\
\frac{\partial c_{b}}{\partial x}=0 \text { on } \Gamma_{\mathrm{tp}} \text { and } \Gamma_{\mathrm{tl}}\left(=\Gamma_{\mathrm{bt}} \cup \Gamma_{\mathrm{st}}\right), \\
c_{f}=0 \text { on } \Gamma_{\mathrm{tp}}, \\
c_{f}=0 \text { or } \frac{\partial c_{\mathrm{f}}}{\partial \mathrm{x}}=0 \text { on } \Gamma_{\mathrm{bt}}, \\
J_{b}(t)=\sqrt{\frac{1}{P e_{c} \pi t}} \text { on } \Gamma_{\mathrm{st}},
\end{gathered}
$$

where $P e_{T}\left[=\left(V_{y} \delta\right) /\left(D_{T}\right)\right]$ and $D a\left[=\left(k_{a} B_{M} \delta^{2}\right) /\left(D_{T}\right)\right]$ are the Peclet number and Damköhler number in the tissue respectively. Here, $\varepsilon_{1}\left(=R_{d} / c_{0}\right)$ and $\varepsilon_{2}\left(=c_{0} / B_{M}\right)$ are two scaling parameters. $P e_{c}\{=$ $\left.\left[V_{y}\left(h^{2} / \delta\right)\right] / D_{c}\right\}$ is the Peclet number in the coating of struts and $h$ is the thickness of the coating of the strut.

\section{Solution procedure}

The governing equations (8-9) representing the transport of free and bound drugs along with the boundary conditions (10-14) are solved numerically by finite-difference scheme. Forward-time centered-space discretization technique is leveraged in this explicit scheme. Following, we describe our finite-difference scheme in more detail. We denote $x_{i}=j \delta x, z_{i}=i \delta z, t^{n}=n \delta t$, where $n$ refers to the time level and $\delta t$ is the time increment. Here, $\delta x$ and $\delta z$ stand for the step sizes along the radial and the axial directions respectively. Steady state is achieved when the convergence criterion for concentration was $10^{-7}$ for both drug forms.

The finite-difference approximation of (8) for the transport of free drug is given by

$$
\frac{c_{f_{i, j}}^{n+1}-c_{f_{i, j}}^{n}}{\delta t}=\left[\operatorname{diff} c_{f}\right]_{i, j}^{n}-[r e a c]_{i, j}^{n},
$$

where $\left[\text { diff } c_{f}\right]_{i, j}^{n}=\left(1 / P e_{T}\right)\left\{\left[\left(\partial^{2} c_{f}\right) /\left(\partial x^{2}\right)\right]_{i, j}^{n}+1 / x_{j}\left[\left(\partial c_{f}\right) /(\partial \mathrm{x})\right]_{i, j}^{n}+\right.$ $\left.\left[\left(\partial^{2} c_{f}\right) /\left(\partial z^{2}\right)\right]_{i, j}^{n}\right\}$ and $[\mathrm{reac}]_{i, j}^{n}=\left(D a / P e_{T}\right)\left[c_{f_{i, j}}\left(1-c_{b_{i, j}}\right)-\varepsilon_{1} c_{b_{i, j}}\right.$.

Likewise, the discretized version of Eq. 9 may be written as

$$
\frac{c_{b_{i, j}}^{n+1}-c_{b_{i, j}}^{n}}{\delta t}=\varepsilon_{2}[\text { reac }]_{i, j}^{n} .
$$

\section{Result and discussion}

For the purpose of numerical computation of the desired quantities of major physiological significance by using the baseline values included in Table $1^{7,13,23,28,33-41}$, solutions are computed through the generation of grids, with a size of $301 \times 101$ for $\delta t=0.00001$. The simulation concerning the grid independence study was performed for the purpose of examining the error associated with the grid sizes used, and is presented in Figure 2a. One may notice from this figure that the transmural variation of normalized free drug concentration concerning the three grid sizes viz $151 \times 51,301 \times$ 101, $601 \times 201$ overlap one another at $t=100$ for $P e_{T}=2$ and $D a$ $=40$. Thus, the grid independence study in the present context of numerical simulation has its own importance to establish the correctness of the results obtained. Figure $2 b$ displays the results of normalized free drug concentration for different time steps $\delta t$ viz $0.0001,0.00001,0.00005$. Here too, the concentration profiles get overlapped for three distinct time steps, which also establish the correctness of the time step used.

Distributions of mean free as well as bound drug concentrations for different values of scaling parameter $\varepsilon_{1}\left(=R_{d} / c_{0}\right)$ are presented in Figure $3 \mathrm{a}$ and $3 \mathrm{~b}$ respectively, and the same for different values of $\varepsilon_{2}\left(=c_{0} / B_{M}\right)$, which are depicted in Figure $4 \mathrm{a}$ and $4 \mathrm{~b}$ respectively. Evidently, $\varepsilon_{1}$, depending on $R_{d}\left(=k_{d} / k_{a}\right)$, decreases with a decrease in the dissociation rate constant $k_{d}$ and also with an increase in the association rate constant $k_{a}$. However, $\varepsilon_{2}$ increases with decreasing $B_{M}$ (keeping $c_{0}$ as fixed). Figure 3 a shows that the normalized mean free drug concentration decreases with decreasing $\varepsilon_{1}$ for $P e_{T}$ $=2, D_{a}=40, \varepsilon_{2}=100$, up to a certain time and, thereafter, no significant changes occurred. It may be justified in the sense that, as $\varepsilon_{1}$ decreases, the rate of reversible binding $\left(k_{d}\right)$ decreases and/or the rate of forward binding increases, which is lowering the mean concentration of free drug.

An interesting phenomenon may be observed when the transported free drug is insensitive to flowing blood [i.e. modelled as zero-flux lumen-tissue interface condition, the mean concentration of free drug is always higher (zoom inset) as compared to zeroconcentration interface condition and approaches a quasi-steady state faster]. Figure $3 \mathrm{~b}$ shows how the rates of forward as well as reversible binding affect the mean concentration of bound drug within the arterial tissue. It is to be observed that the mean concentration is increased with the decrease of $\varepsilon_{1}$, owing to the increase in the rate of forward binding and/or to the decrease in the rate of reversible binding. Here too, the mean concentration of bound drug appears to be higher in case of zero-flux lumen-tissue interface condition, and saturation of binding sites takes place very rapidly.

Effects of $\varepsilon_{2}$ (i.e. net tissue binding potential on the mean concentrations of free as well as bound drug) are displayed in Figure $4 \mathrm{a}$ and $4 \mathrm{~b}$ respectively. It may be recalled that $\varepsilon_{2}$ increases with 
Table 1. Plausible values of involved parameters

\begin{tabular}{|c|c|c|c|}
\hline Description & Parameter & Value & Reference \\
\hline Strut dimension, $\mathrm{m}$ & $\delta$ & 0.0001 & [13] \\
\hline Strut coating thickness, m & $h$ & $5.0 \times 10^{-5}$ & [33] \\
\hline Mean wall thickness, m & $A w(=10 \delta)$ & 0.001 & [34] \\
\hline Interstrut distance, $\mathrm{m}$ & $\Delta(=7 \delta)$ & 0.0007 & [35] \\
\hline Transmural filtration velocity, $\mathrm{m} \mathrm{s}^{-1}$ & $V_{y}$ & $4 \times 10^{-8}$ & [36] \\
\hline Porosity of the arterial wall & $\varepsilon$ & 0.787 & [37] \\
\hline Tortuosity of the arterial wall & $\tau$ & 1.333 & [37] \\
\hline Coating drug diffusivity, $\mathrm{m}^{2} \mathrm{~s}^{-1}$ & $D_{c}$ & $1 \times 10^{-15}$ & [38] \\
\hline Coefficient of free diffusivity, $\mathrm{m}^{2} \mathrm{~s}^{-1}$ & $D_{\text {free }}$ & $3.65 \times 10^{-12}$ & {$[28,39]$} \\
\hline Coefficient of effective diffusivity, $\mathrm{m}^{2} \mathrm{~s}^{-1}$ & $D_{e f f}$ & $2.15 \times 10^{-12}$ & Our study \\
\hline True diffusivity of the free drug, $\mathrm{m}^{2} \mathrm{~s}^{-1}$ & $D_{T}$ & $24 \times 10^{-12}$ & Our study \\
\hline Initial drug concentration in the coating, $\mathrm{mol} \mathrm{m}^{-3}$ & $c_{0}$ & 1 & [23] \\
\hline Net tissue binding capacity, $\mathrm{mol} \mathrm{m}^{-3}$ & $B_{M}$ & 0.01 & [7] \\
\hline Association rate constant, $\mathrm{m}^{3} \mathrm{~mol}^{-1} \mathrm{~s}^{-1}$ & $k_{a}$ & 10 & [40] \\
\hline Dissociation rate constant, $\mathrm{s}^{-1}$ & $k_{d}$ & 0.01 & [41] \\
\hline Equilibrium dissociation constant, $\mathrm{mol} \mathrm{m}^{-3}$ & $R_{d}$ & 0.001 & Our study \\
\hline Dimensionless Peclet number in the coating & $P e_{c}$ & 100 & Our study \\
\hline Dimensionless Peclet number in the tissue & $P e_{T}$ & 2 & Our study \\
\hline Dimensionless Damköhlar number in the tissue & $D a$ & 40 & Our study \\
\hline Dimensionless scaling parameter & $\varepsilon_{1}$ & 0.001 & Our study \\
\hline Dimensionless scaling parameter & $\varepsilon_{2}$ & 100 & Our study \\
\hline
\end{tabular}

decreasing binding potential. The results of these figures indicate that the mean concentration of free drug increases with decreasing binding potential up. to $\varepsilon_{2}=100$, but the concentration reaches a quasi-steady state for weaker binding capacity $\left(\varepsilon_{2}=1,000\right)$ than the other cases. As anticipated, the mean concentration of free drug appears to be higher for zero-flux lumen-tissue interface condition (zoom inset) (Fig. 4a). Similar observations can be made for normalized bound drug concentration but with a distinction. In the case of that free drug for $\varepsilon_{2} \leq 100$, the quasi-equilibrium is not fully established until $t=500$. On the other hand, in the case that bound drug for $\varepsilon_{2} \geq 10$, the quasi-equilibrium is attained very rapidly (Fig. 4b). Furthermore, the effects of zero-flux lumen-tissue interface condition on the spatiotemporal distribution of free and bound drug can be visualized in Figure 5, panels a and $b$.

The time-evolution of the normalized free and bound drug concentrations at positions $P_{1}$ and $P_{2}$ (near the left and the perivascular
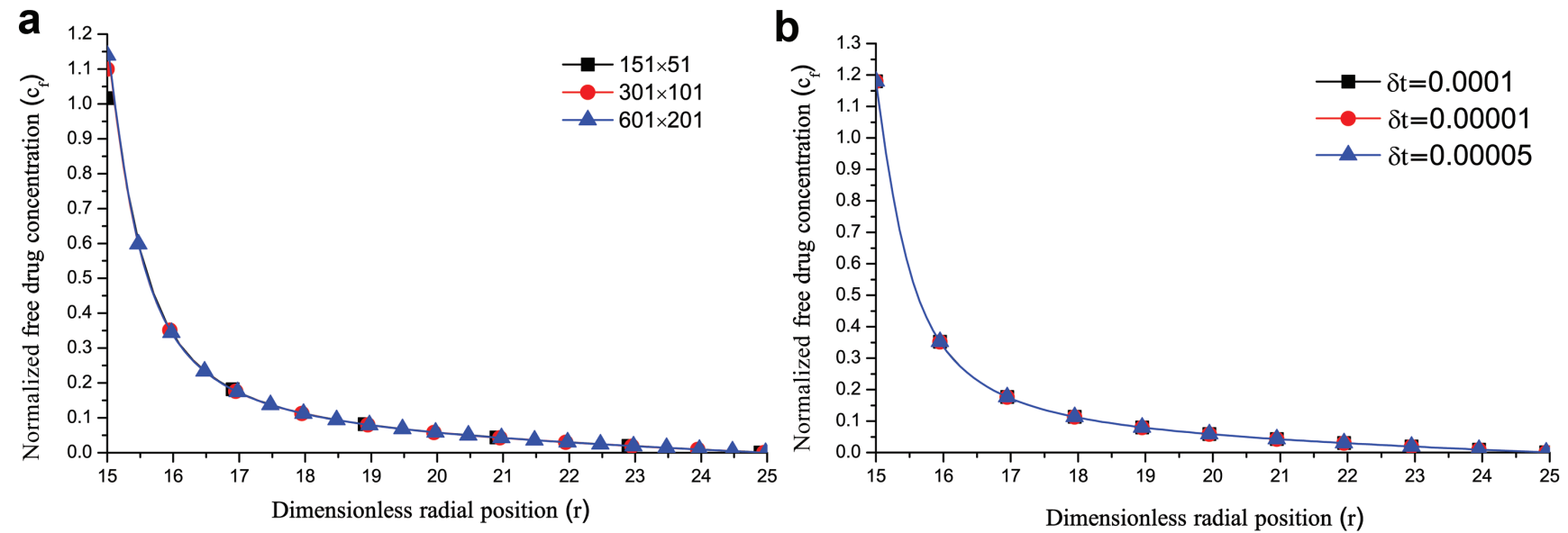

Fig. 2. Transmural variation of normalized free drug concentration for $\boldsymbol{P} \boldsymbol{e}_{T}=\mathbf{2}, \boldsymbol{D a}=\mathbf{4 0}$ and $\boldsymbol{t}=\mathbf{1 0 0}$ at $\boldsymbol{z}=\mathbf{1 1}$. a: Different grid sizes; $\mathrm{b}$ : different time steps. 

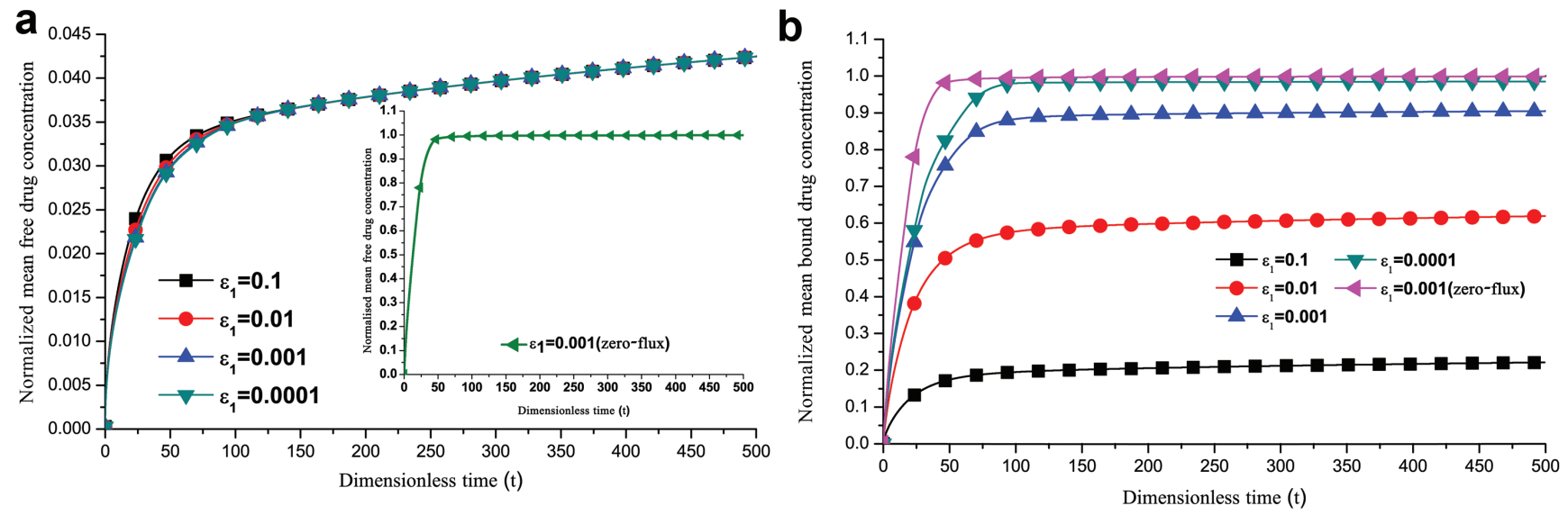

Fig. 3. Distribution of normalized mean drug concentration for different values of $\varepsilon_{1}$ at $P e_{T}=2, D a=40, \varepsilon_{2}=100$ and $t=500$. a: Free drug; b: bound drug.
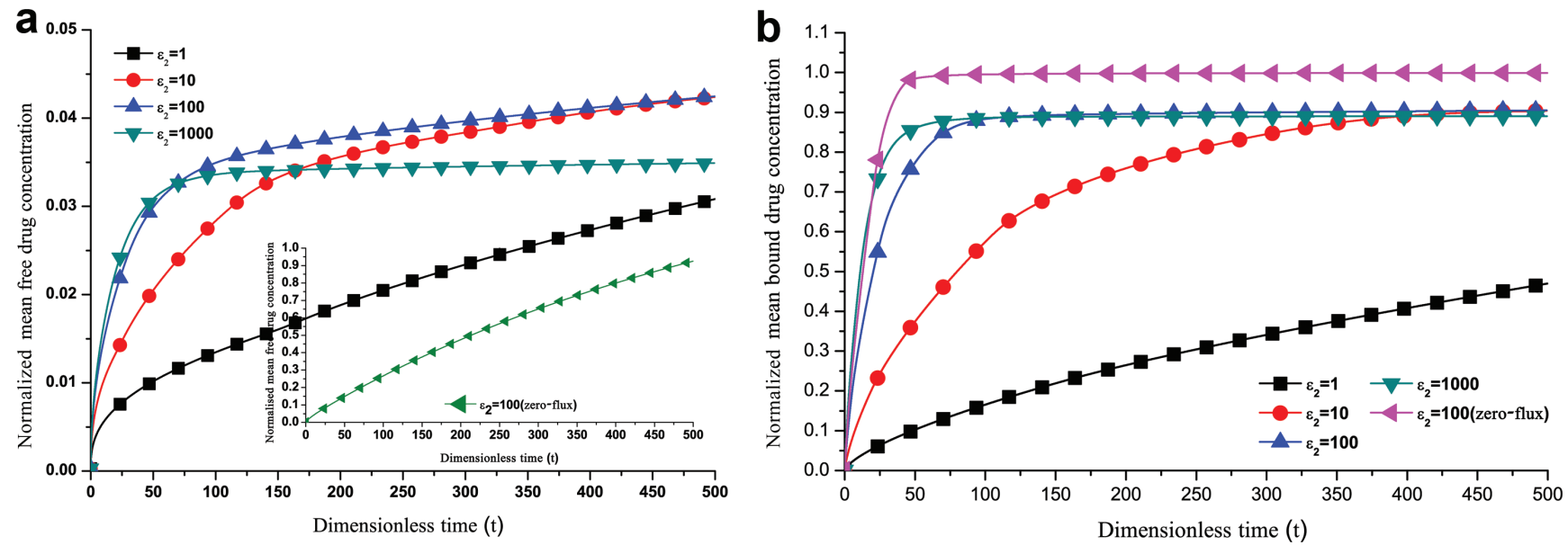

Fig. 4. Distribution of normalized mean drug concentration for different values of $\varepsilon_{2}$ at $P e_{T}=2, D a=40, \varepsilon_{1}=0.001$ and $t=500$. a: Free drug; b: bound drug.

boundaries in Fig. 1) are shown in Figure 6, panels a, b and c, $\mathrm{d}$ respectively. The drug distribution profiles indicate that quasiequilibrium has not yet been fully established until $t=500$ for small values of $D a$ and also that the normalized free drug concen-

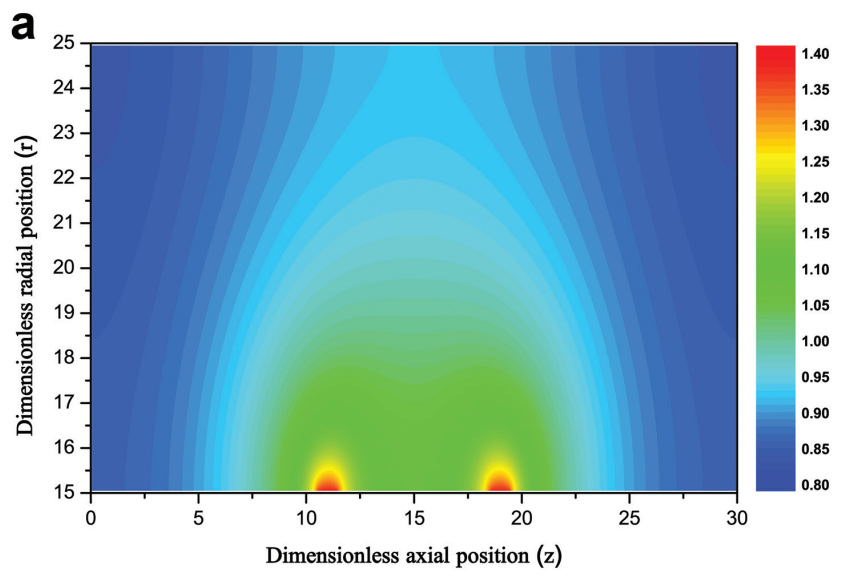

tration at $P_{1}$ and $P_{2}$ decreases with increasing $D a$ (Fig. 6a and 6b). Moreover, the reduced local free drug concentration at $P_{2}$ with increasing $D a$ further reduces the drug dissipation at the perivascular boundary. Similar results on the distribution of bound drug have

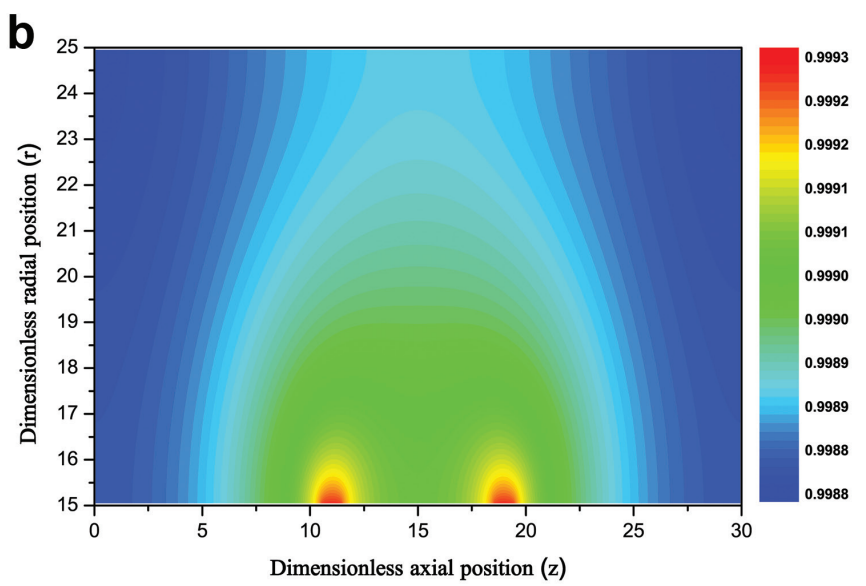

Fig. 5. Visual representation of drug concentration at $P e_{T}=2, D a=40$ and $t=500$. a: Free drug $\left(J_{b}=0\right.$ on $\Gamma_{b t}$ for $\left.c_{f}\right) ; b$ : bound drug $\left(J_{b}=0\right.$ on $\Gamma_{b t}$ for $\left.c_{f}\right)$. 

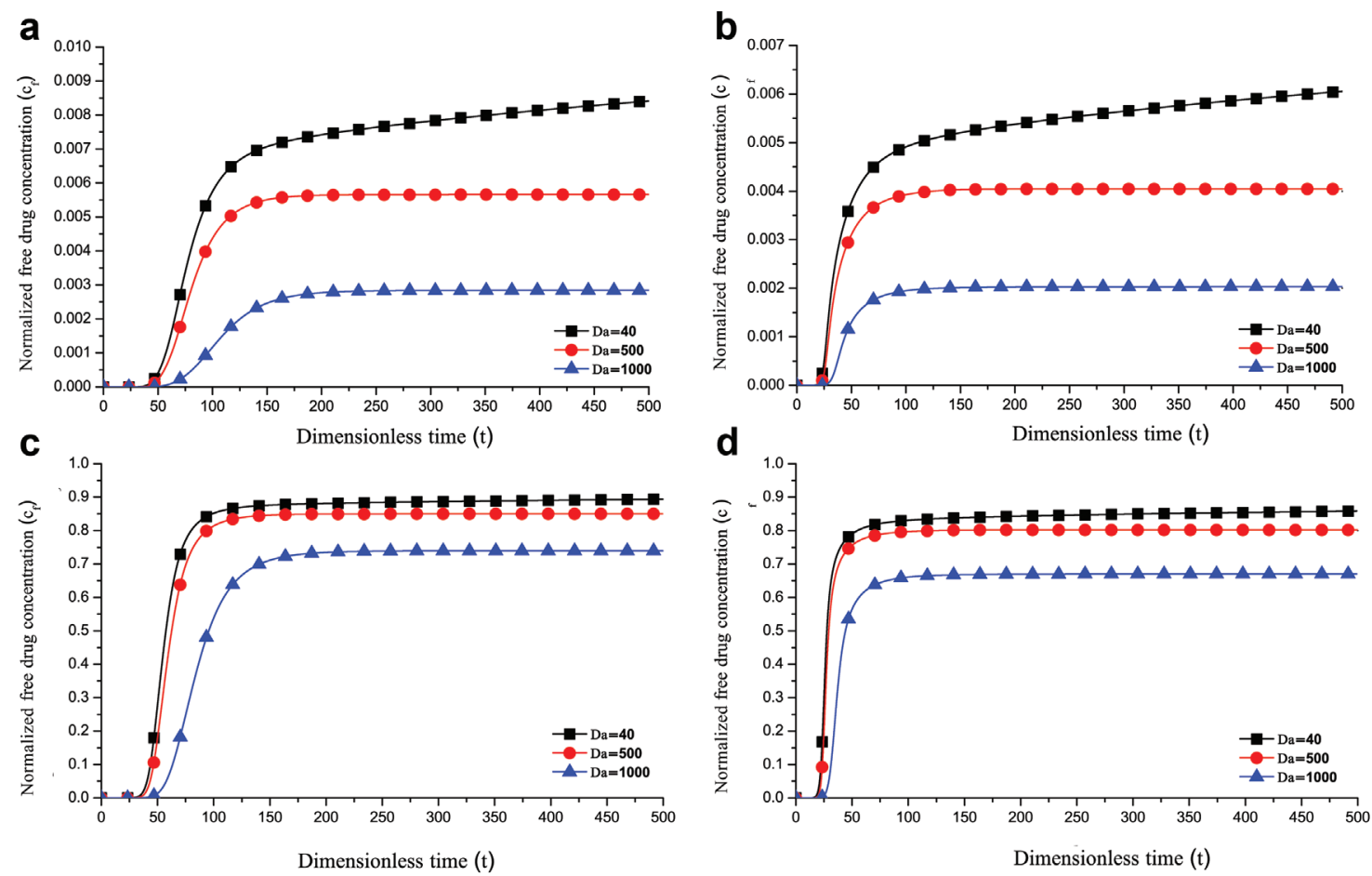

Fig. 6. Temporal variation of normalized drug concentration at different positions of the domain for different $D a$ at $P e_{T}=\mathbf{2}$ and $t=500$. a: Free drug at $\mathrm{P}_{1}$; $b$ : free drug at $\mathrm{P}_{2}$, bound drug at $\mathrm{P}_{1}$; $\mathrm{d}$ : bound drug at $\mathrm{P}_{2}$.
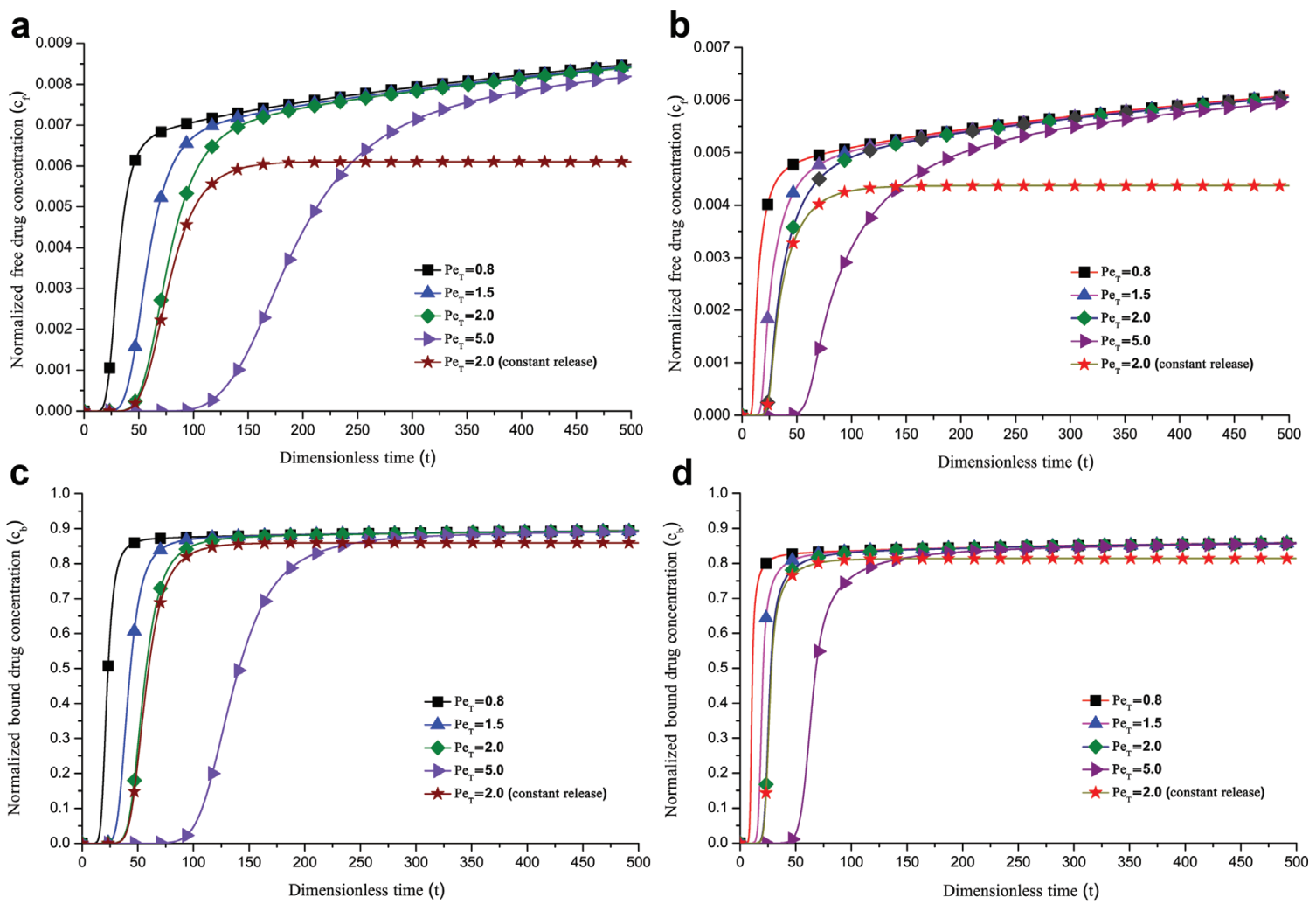

Fig. 7. Temporal variation of normalized drug concentration at different positions of the domain for different $P e_{T}$ at $D a=40$ and $t=500$. a: Free drug at $\mathrm{P}_{1} ; \mathrm{b}$ : free drug at $\mathrm{P}_{2}, c$ bound drug at $\mathrm{P}_{1} ; \mathrm{d}$ : bound drug at $\mathrm{P}_{2}$. 
a
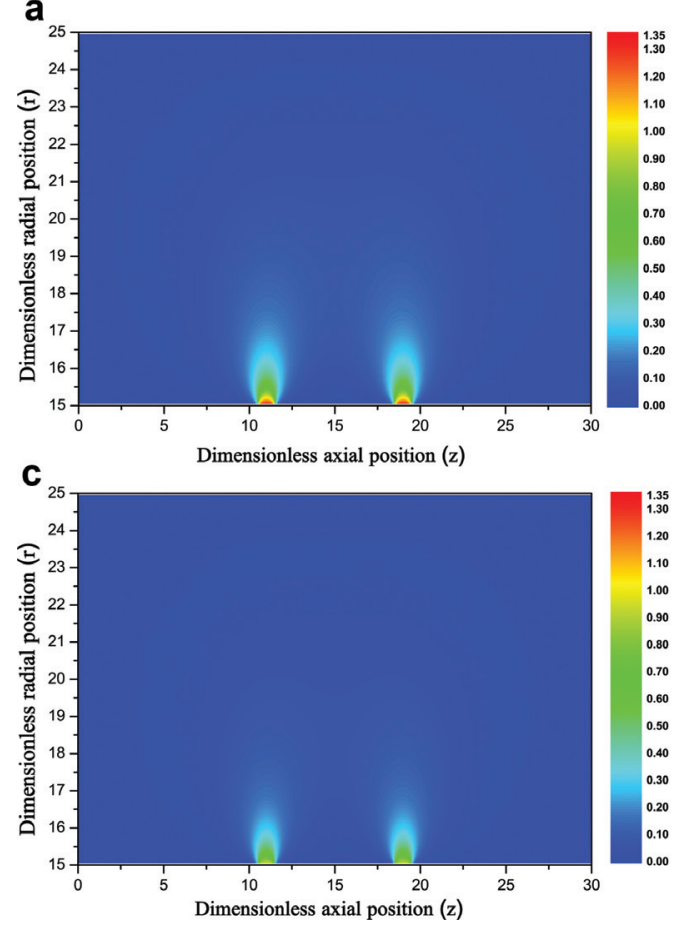

b

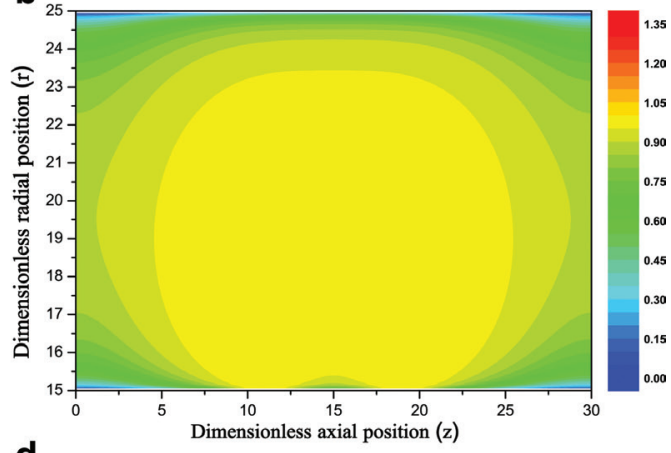

d

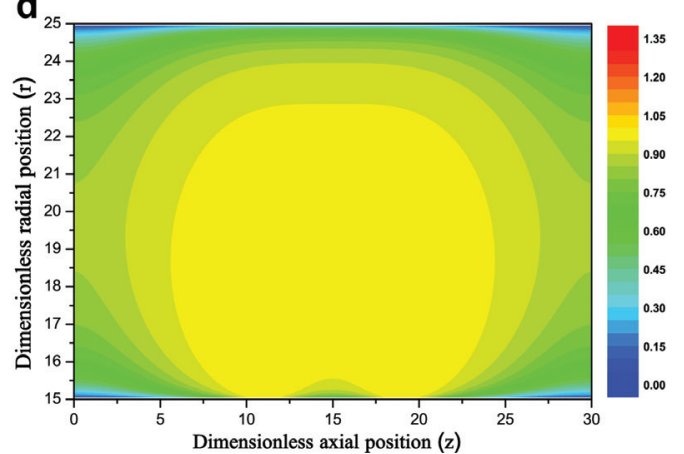

Fig. 8. Visual representation of drug concentration at $\mathbf{P} \boldsymbol{e}_{T}=\mathbf{2}, \mathbf{D a}=\mathbf{4 0}$ and $\boldsymbol{t}=\mathbf{5 0 0}$. a: Free (time-dependent release); b: bound (time-dependent release); c: free (constant release); $d$ : bound (constant release).

been shown in Figure 6c and 6d. All of the above observations are in good agreement with those of Hwang et al. ${ }^{15}$ who studied the distribution of free drug only. The lack of drug far away from the strut (i.e. at $P_{2}$ ) can be a serious factor for in-stent restenosis occurrence, as high drug concentration at the perivascular end is more important in suppressing restenosis. ${ }^{42-44}$

The influences of diffusivity (Peclet number) and time-dependent release kinetics of a drug on the temporal variation of free as well as bound drug concentrations within the arterial tissue are portrayed in Figure 7, panels a, b and c, d respectively. Figure $7 \mathrm{a}$ and $7 \mathrm{~b}$ indicate that the free drug concentrations at both $P_{1}$ and $P_{2}$ do not attain quasi-equilibrium state until $t=500$, due to the time-dependent release-kinetics. However, if one assumes

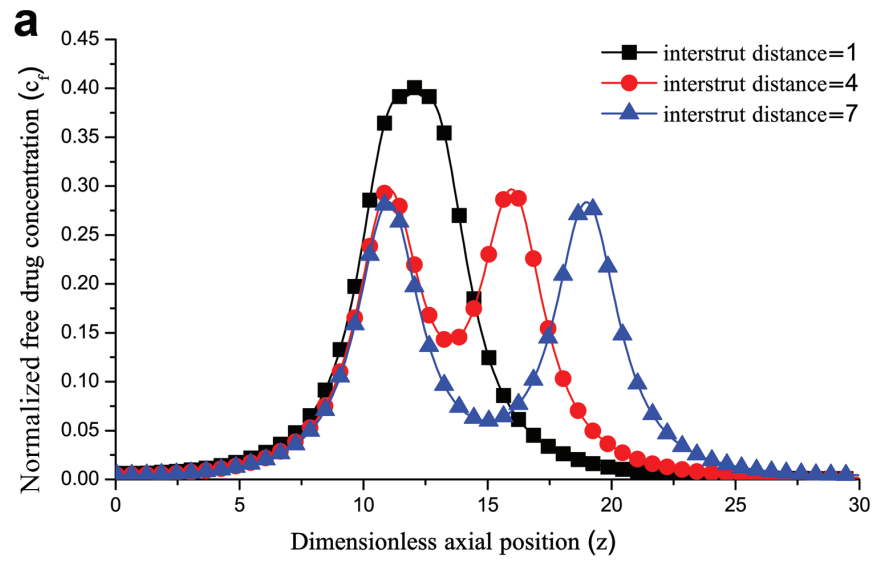

constant release of drug from a well apposed strut, a quasi-steady state has been fully established at the positions considered. On the contrary, the bound drug concentration attains a quasi-steady state irrespective of time-dependent and constant release of drug. The effect of release kinetics on the spatial distribution of free as well as bound drug can also be visualized clearly in Figure 8, panels $\mathrm{a}-\mathrm{d}$, in which heterogeneous distribution and retention of drug are found to be observed throughout the domain and higher concentration of free drug is observed in case of time-dependent release of drug from struts.

Figure 9a shows the concentration profile at a height of one strut thickness for free drug concentration depending on interstrut distance for $P e_{T}=2$ and $D a=40$ at $t=500$. A single peak is noted

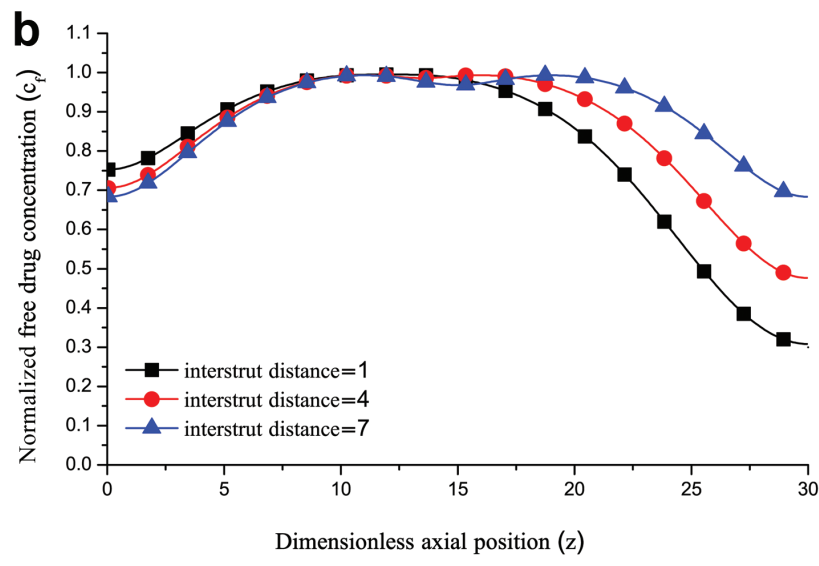

Fig. 9. Distribution of normalized drug concentration for various interstrut distances at one-strut height within tissue for $P e_{T}=2, D a=40$ and $t=500$. a: Free drug; b: bound drug. 

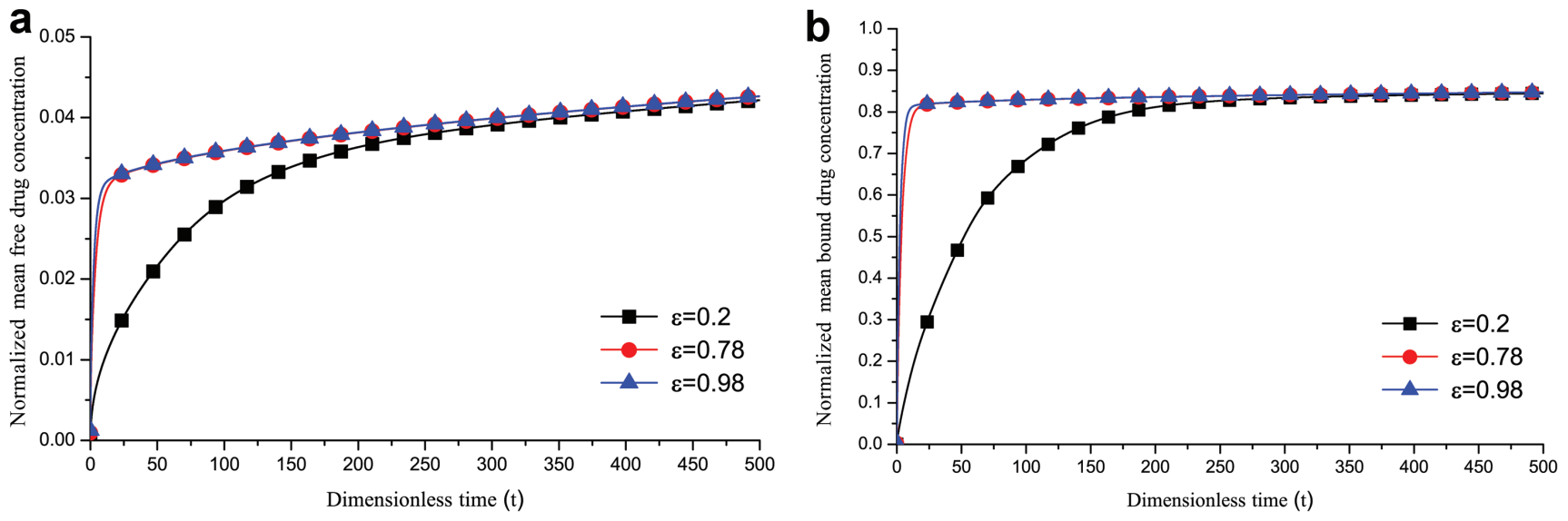

Fig. 10. Temporal variation of normalized mean drug concentration for different porosities $(\varepsilon)$ at $P e_{T}=2, D a=40$ and $t=500$. a: Free drug; b: bound drug.

when the struts are placed one-strut width apart. As the interstrut distance increases, the peak concentration falls and distinct peaks over each strut are observed. The distributions of bound drug concentration are analogous to those of the distributions of free drug qualitatively (Fig. 9b).

When a stent is implanted within an artery, it has a major impact on the structures of the wall and subsequently alters the transport and retention of the drug within the arterial tissue. Any porous material under compression will demonstrate smaller pore ratio than that of its relaxed state, which in turn influences the tortuosity. Arterial property such as porosity $(\varepsilon)$ dictates the transport and retention of drug eluted from a pair of struts, as depicted in Figure $10 \mathrm{a}$ and $10 \mathrm{~b}$ respectively. It is observed from these figures that the mean concentrations of free as well as bound drug increases with increasing $\varepsilon$. However, the enhancement is maximum when $\varepsilon$ is allowed to change from 0.2 to 0.78 , up to $t=250$ for both drug forms. It is interesting to note that the mean concentration of bound drug attains a quasi-steady state for larger $\varepsilon$ (i.e. saturation of binding sites takes places very rapidly).

The above observation may be justified in the sense that as the porosity increases, the effective as well as true diffusivity do increase, which eventually leads to expedition of the diffusion process. In a porous media, diffusion takes place in confined tortuous pores and its progression is impeded as the tortuosity increases. Our simulation also demonstrates the fact that a decrease in the mean concentration of free drug with increasing tortuosity $(\tau)$ (Fig.

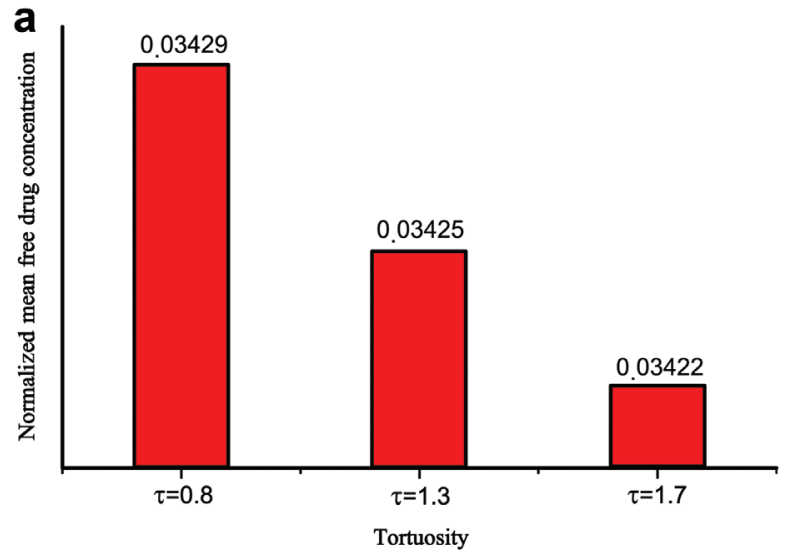

11a) (i.e. an inverse relationship between free drug concentration and tortuosity is revealed). A similar pattern is also observed for bound drug (Fig. 11b). The above observation may be justified in the sense that as the tortuosity increases so too does the effective distance over which diffusion has to take place (i.e. the progression of diffusion eventually lowering the mean concentration of both drug forms is impeded).

\section{Conclusions and future work}

In this numerical study, a two-dimensional axisymmetric model of drug transport eluted from drug-eluting stents has been developed. We consider a two-species model, capable of predicting the delivery of drug and its retention in the arterial tissue where the transport of free drug is governed by reaction-diffusion process and that of bound drug, assuming complete immobilization in the tissue, by reaction process. Following Tzafriri et al. ${ }^{19}$ a second-order dynamic model that describes a saturating reversible binding process has been taken into account. A time-dependent release kinetic is applied on the surface of the struts. ${ }^{20,23}$ As the arterial structures such as porosity and tortuosity affect the diffusion process, their influence on the transport and retention of drug eluted from a pair of struts has been investigated successfully.

Though experimental studies can provide information on release kinetics and other histological information, computational

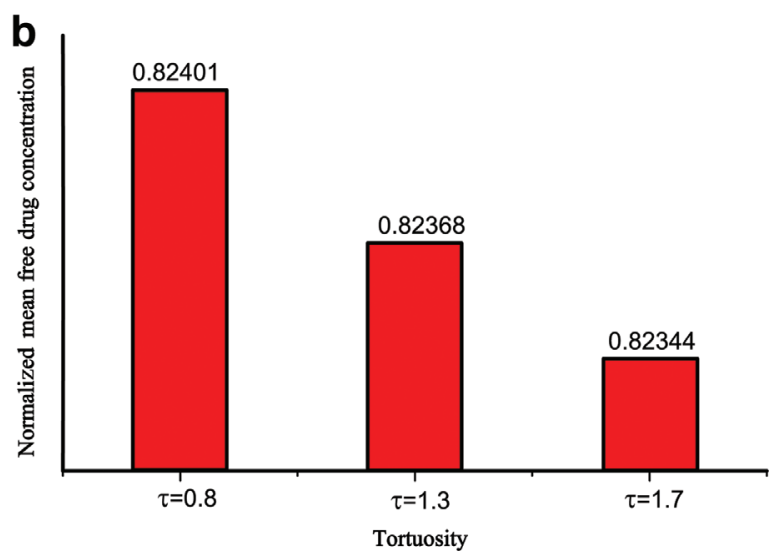

Fig. 11. Normalized mean drug concentration for varying tortuosity $(\tau)$ at $P e_{T}=2, D a=40$ and $t=500$. a: Free drug; b: bound drug. 
studies can provide detailed predictions of the drug distribution and its retention within the arterial tissue. With the rapid ascent of stent-based drug delivery in the treatment of vascular disease, many important issues concerning drug delivery and its retention in the arterial tissue need to be addressed. Indeed, arterial vessels with disease are the target sites for endovascular intervention, and quantifying the pharmacokinetics for this scenario is needed towards full appreciation of drug-eluting stents and like technologies.

\section{Acknowledgments}

The authors gratefully acknowledge the careful scrutiny and suggestions of the learned reviewers. The authors gratefully acknowledge the partial financial support from Special Assistance Programme (SAP-III) sponsored by University Grants Commission (UGC), New Delhi, India (Grant No. F.510/3/DRS-III/2015(SAPI)).

\section{Conflict of interest}

The authors have no conflict of interests related to this publication.

\section{Author contributions}

Formulating the problem (RS, PKM); running the in-house code (RS); writing the manuscript (RS, PKM).

\section{References}

[1] Balakrishnan B, Dooley J, Kopia G, Edelman ER. Thrombus causes fluctuations in arterial drug delivery from intravascular stents. J Control Release 2008;131(3):173-180. doi:10.1016/j.jconrel.2008.07.027.

[2] Balakrishnan B, Tzafriri AR, Seifert P, Groothuis A, Rogers C, Edelman ER. Strut position, blood flow, and drug deposition implications for single and overlapping drug-eluting stents. Circulation 2005;111(22):29582965. doi:10.1161/CIRCULATIONAHA.104.512475.

[3] Costa MA, Simon DI. Molecular basis of restenosis and drug-eluting stents. Circulation 2005;111(17):2257-2273. doi:10.1161/01. CIR.0000163587.36485.A7.

[4] Daemen J, Serruys PW. Drug-eluting stent update 2007 part I. A survey of current and future generation drug-eluting stents: meaningful advances or more of the same? Circulation 2007;116(3):316-328. doi:10.1161/CIRCULATIONAHA.106.621342.

[5] Hose D, Narracott A, Griffiths B, Mahmood S, Gunn J, Sweeney D, et al. A thermal analogy for modelling drug elution from cardiovascular stents. Comput Methods Biomech Biomed Engin 2004;7(5):257-264. doi:10.1080/10255840412331303140.

[6] Hwang CW, Levin AD, Jonas M, Li PH, Edelman ER. Thrombosis modulates arterial drug distribution for drug-eluting stents. Circulation 2005;111(13):1619-1626. doi:10.1161/01.CIR.0000160363.30639.37.

[7] Levin AD, Vukmirovic N, Hwang CW, Edelman ER. Specific binding to intracellular proteins determines arterial transport properties for rapamycin and paclitaxel. Proc Natl Acad Sci U S A 2004;101(25):94639467. doi:10.1073/pnas.0400918101.

[8] McGinty S, McKee S, Wadsworth RM, McCormick C. Modelling drugeluting stents. Math Med Biol 2011;28(1):1-29. doi:10.1093/imam$\mathrm{mb} / \mathrm{dqq003.}$

[9] McGinty S, McKee S, Wadsworth RM, McCormick C. Modeling arterial wall drug concentrations following the insertion of a drug-eluting stent. SIAM J Appl Math 2014;73(6):2004-2028. doi:10.1137/ 12089065X.
[10] Lovich MA, Philbrook M, Sawyer S, Weselcouch E, Edelman ER. Arterial heparin deposition: role of diffusion, convection, and extravascular space. Am J Physiol 1998;275(6 Pt 2):H2236-H2242.

[11] Moses JW, Stone GW, Nikolsky E, Mintz GS, Dangas G, Grube E, et al. Drug-eluting stents in the treatment of intermediate lesions: pooled analysis from four randomized trials. J Am Coll Cardiol 2006;47(11):2164-2171. doi:10.1016/j.jacc.2006.01.068.

[12] Serruys PW, Sianos G, Abizaid A, Aoki J, den Heijer P, Bonnier $H$, et $a l$. The effect of variable dose and release kinetics on neointimal hyperplasia using a novel paclitaxel-eluting stent platform: the paclitaxel in-stent controlled elution study (pisces). J Am Coll Cardiol 2005;46(2):253-260. doi:10.1016/j.jacc.2005.03.069.

[13] Lovich MA, Edelman ER. Computational simulations of local vascular heparin deposition and distribution. Am J Physiol 1996;271(5 Pt 2):H2014-H2024. doi:10.1152/ajpheart.1996.271.5.H2014.

[14] Sakharov DV, Kalachev LV, Rijken DC. Numerical simulation of local pharmacokinetics of a drug after intravascular delivery with an eluting stent. J Drug Target 2002;10(6):507-513. doi:10.1080/10611860 21000038382.

[15] Hwang C, Wu D, Edelman E. Physiological transport forces govern drug distribution for stent-based delivery. Circulation 2001;104:600605. doi:10.1161/hc3101.092214.

[16] Migliavacca F, Gervaso F, Prosi M, Zunino P, Minisini S, Formaggia L, et al. Expansion and drug elution model of a coronary stent. Comput Methods Biomech Biomed Engin 2007;10(1):63-73. doi:10.1080/ 10255840601071087.

[17] Borghi A, Foa E, Balossino R, Migliavacca F, Dubini G. Modelling drug elution from stents: effects of reversible binding in the vascular wall and degradable polymeric matrix. Comput Methods Biomech Biomed Engin 2008;11(4):367-377. doi:10.1080/10255840801887555.

[18] Horner M, Joshi S, Dhruva V, Sett S, Stewart S. A two-species drug delivery model is required to predict deposition from drug-eluting stents. Cardiovascular Engineering and Technology 2010;1(3):225234. doi:10.1007/s13239-010-0016-4.

[19] Tzafriri AR, Levin AD, Edelman ER. Diffusion-limited binding explains binary dose response for local arterial and tumour drug delivery. Cell Prolif 2009;42(3):348-363. doi:10.1111/j.1365-2184.2009.00602.x.

[20] Higuchi T. Mechanism of sustained-action medication. theoretical analysis of rate of release of solid drugs dispersed in solid matrices. J Pharm Sci 1963;52(12):1145-1149. doi:10.1002/jps.2600521210.

[21] Mandal AP, Sarifuddin, Mandal PK. An unsteady analysis of arterial drug transport from half-embedded drug-eluting stent. Appl Math Comput 2015;266:968-981. doi:10.1016/j.amc.2015.06.033.

[22] Mandal AP, Mandal PK. Computational modelling of three-phase stent-based delivery. J Explor Res Pharmacol 2017;2:31-40. doi:10.14218/JERP.2017.00001.

[23] O'Brien C, Kolachalama V, Barber T, Simmons A, Edelman E. Impact of flow pulsatility on arterial drug distribution in stent-based therapy. J Control Release 2013;168(2):115-124. doi:10.1016/j.jconrel.2013.03.014.

[24] Saha R, Sarifuddin, Misra JC, Mandal PK. Impact of luminal flow on mass transport through coronary arteries: a study relevant to drugeluting stent. Int J Math Comput 2016;27(3):40-58.

[25] Saltzman WM. Drug delivery: engineering principles for drug therapy. Oxford University Press, USA, 2001.

[26] Satterfield CN. Mass transfer in heterogeneous catalysis. The MIT Press, 1970.

[27] O'Connell BM, Walsh MT. Demonstrating the influence of compression on artery wall mass transport. Ann Biomed Eng 2010;38(4):13541366. doi:10.1007/s10439-010-9914-8.

[28] Kolachalama VB, Levine EG, Edelman ER. Luminal flow amplifies stent-based drug deposition in arterial bifurcations. PLoS One 2009; 4(12):e8105. doi:10.1371/journal.pone.0008105.

[29] Sarifuddin, Mandal PK. Effect of diffusivity on the transport of drug eluted from drug-eluting stent. Int J Appl Comput Math 2016;2(2):291-301. doi:10.1007/s40819-015-0060-8.

[30] Kolandaivelu K, O'Brien CC, Shazly T, Edelman ER, Kolachalama VB. Enhancing physiologic simulations using supervised learning on coarse mesh solutions. J R Soc Interface 2015;12(104):20141073. doi:10.1098/rsif.2014.1073.

[31] PontrelliG, DeMonteF.Modeling ofmass dynamicsinarterialdrug-eluting stents. Journal of Porous Media 2009;12(1):19-28. doi:10.1615/ 
J Explor Res Pharmacol

JPorMedia.v12.i1.20.

[32] Kolachalama VB, Pacetti SD, Franses JW, Stankus JJ, Zhao HQ, Shazly $\mathrm{T}$, et al. Mechanisms of tissue uptake and retention in zotarolimus-coated balloon therapy. Circulation 2013;127(20):2047-2055. doi:10.1161/CIRCULATIONAHA.113.002051.

[33] Mongrain R, Faik I, Leask RL, Rodes-Cabau J, Larose E, Bertrand OF. Effects of diffusion coefficients and struts apposition using numerical simulations for drug eluting coronary stents. J Biomech Eng 2007;129(5):733-742. doi:10.1115/1.2768381.

[34] Moore JE, Ku DN. Pulsatile velocity measurements in a model of the human abdominal aorta under resting conditions. J Biomech Eng 1994;116(3):337-346. doi:10.1115/1.2895740.

[35] Vairo G, Cioffi M, Cottone R, Dubini G, Migliavacca F. Drug release from coronary eluting stents: a multidomain approach. J Biomech 2010;43(8):1580-1589. doi:10.1016/j.jbiomech.2010.01.033.

[36] Khakpour M, Vafai K. Critical assessment of arterial transport models. Int J Heat Mass Transf 2008;51(3):807-822. doi:10.1016/j.ijheatmasstransfer.2007.04.021.

[37] O'Connell BM, Walsh MT. Arterial mass transport behaviour of drugs from drug eluting stents. In: Ghista DN, editor. Biomedical Science, Engineering and Technology. London: InTechOpen; 2012. p. 301-324.

[38] Kamath KR, Barry JJ, Miller KM. The taxus drug-eluting stent: A new paradigm in controlled drug delivery. Adv Drug Deliv Rev
Saha R. et al: Release kinetics in stent-based drug delivery

2006;58(3):412-436. doi:10.1016/j.addr.2006.01.023.

[39] Creel CJ, Lovich MA, Edelman ER. Arterial paclitaxel distribution and deposition. Circ Res 2000;86(8):879-884. doi:10.1161/01. RES.86.8.879.

[40] Zhang F, Fath M, Marks R, Linhardt RJ. A highly stable covalent conjugated heparin biochip for heparin-protein interaction studies. Anal Biochem 2002;304(2):271-273. doi:10.1006/abio.2002.5617.

[41] Zhu X, Pack DW, Braatz RD. Modelling intravascular delivery from drug-eluting stents with biodurable coating: investigation of anisotropic vascular drug diffusivity and arterial drug distribution. Comput Methods Biomech Biomed Engin 2014;17(3):187-198. doi:10.1080/ 10255842.2012.672815.

[42] Bozsak F, Chomaz JM, Barakat Al. Modeling the transport of drugs eluted from stents: physical phenomena driving drug distribution in the arterial wall. Biomech Model Mechanobiol 2014;13(2):327-347. doi:10.1007/s10237-013-0546-4.

[43] McGinty S, McKee S, McCormick C, Wheel M. Release mechanism and parameter estimation in drug-eluting stent systems: analytical solutions of drug release and tissue transport. Math Med Biol 2015;32(2):163-186. doi:10.1093/imammb/dqt025.

[44] Wessely R, Schömig A, Kastrati A. Sirolimus and paclitaxel on polymer-based drug-eluting stents: similar but different. J Am Coll Cardiol 2006;47(4):708-714. doi:10.1016/j.jacc.2005.09.047. 